\title{
Descarte de resíduos infectantes: informações demonstradas e ações praticadas por estudantes de enfermagem e medicina
}

\section{Disposal of infective waste: demonstrated information and actions taken by nursing and medical students}

\author{
Descarte de desechos infecciosos: informaciones demostradas y acciones practicadas
} por estudiantes de enfermería y medicina

Adenícia Custodia Silva Souza ${ }^{1}$, Sergiane Bisinoto Alves ${ }^{2}$, Mary Rocha Carneiro Garcia Zapata ${ }^{3}$, Anaclara Ferreira Veiga Tipple ${ }^{4}$, Larissa Oliveira Rocha ${ }^{5}$, Janaína Valadares Guimarães ${ }^{6}$, Milca Severino Pereira ${ }^{7}$ ${ }^{1}$ Enfermeira, Doutora em Enfermagem. Professora Adjunta da Pontifícia Universidade Católica de Goiás (PUC-GOIÁS). Goiânia, GO, Brasil. E-mail: adeniciafen@gmail.com.

2 Enfermeira, Doutora em Enfermagem. Enfermeira da Coordenação Municipal de Controle de Infecção em Serviços de Saúde de Goiânia. Goiânia, Go, Brasil. E-mail: sergianebisinoto@yahoo.com.br.

${ }^{3}$ Enfermeira, Mestre em Enfermagem. Presidente da Comissão de Controle de Infecção Hospitalar do Hospital das Clínicas da Universidade Federal de Goiás (UFG). Goiânia, GO, Brasil. E-mail: maryrocar@yahoo.com.br.

${ }^{4}$ Enfermeira, Doutora em Enfermagem. Professora Adjunta da Faculdade de Enfermagem (FEN) da UFG. Goiânia, Go, Brasil. E-mail: anaclara@fen.ufg.br.

${ }^{5}$ Enfermeira, Mestre em Enfermagem. Enfermeira da Secretaria Municipal de Saúde de Goiânia. Goiânia, GO, Brasil. E-mail: larisssarocha@yahoo.com.br.

${ }^{6}$ Enfermeira, Doutora em Enfermagem. Professora Associada da FEN/UFG. Goiânia, GO, Brasil. E-mail: guimaraesjv@hotmail.com.

${ }^{7}$ Enfermeira, Doutora em Enfermagem. Professora Adjunta da PUC-GOIÁS. Goiânia, GO, Brasil. E-mail: milcaseverino@gmail.com.

\section{RESUMO}

O descarte inadequado de resíduos infectantes gera riscos ocupacionais e ambientais e representa a principal causa de acidentes com material biológico. O presente estudo objetivou verificar o conhecimento sobre o descarte de resíduos infectantes e a prática dele entre acadêmicos dos cursos de enfermagem e de medicina de uma universidade pública de Goiás. Os dados foram coletados por meio da aplicação de um questionário. Os estudantes respondentes foram observados na prática e os dados registrados em check list. Os acadêmicos de enfermagem apresentaram maior conhecimento que os de medicina sobre o descarte de luvas contaminadas ( $x^{2}$; $p<0,001)$, bem como sobre o descarte de perfurocortantes $(p=0,001)$. O descarte de luvas contaminadas foi feito em saco para resíduos comum, tanto pelos graduandos de enfermagem quanto pelos de medicina. Os resultados evidenciaram que o conhecimento dos estudantes sobre descarte de resíduos infectantes mostrou-se frágil e insuficiente para garantir a sua aplicabilidade na prática.

Descritores: Resíduos de Serviços de Saúde; Gerenciamento de Resíduos; Estudantes de Enfermagem; Estudantes de Medicina; Exposição Ocupacional.

\section{ABSTRACT}

The inappropriate disposal of infectious waste generates occupational and environmental risks, representing the main cause of accidents with biological material. The aim of the present study was to verify the knowledge and the practice regarding the disposal of infectious waste among nursing and medical undergraduate students at a public university in the state of Goiás. Data were collected with the application of a questionnaire. The respondent students were observed in their practice and data were recorded in a checklist. Nursing students presented greater knowledge than medical students on the disposal of contaminated gloves $\left(x^{2} ; p<0.001\right)$, as well as on the disposal of sharp cutting instruments $(p=0.001)$. Contaminated gloves were disposed of into bags for common waste both by the nursing and the medical students. Results evidenced that the knowledge of students on the disposal of infectious waste was poor and insufficient to ensure its application to practice.

Descriptors: Medical Waste; Waste Management; Students, Nursing; Students, Medical; Occupational Exposure.

\section{RESUMEN}

El descarte incorrecto de residuos infecciosos genera riesgos laborales y ambientales, representando la principal causa de accidentes con material biológico. Se objetivó verificar el conocimiento y la práctica sobre descarte de desechos infecciosos entre estudiantes de grado de enfermería y medicina de una universidad pública de Goiás. Datos recolectados mediante aplicación de cuestionario. Los participantes fueron observados en la práctica, y los datos se registraron en check list. Los estudiantes de enfermería presentaron mayores conocimientos que los de medicina sobre descarte de guantes contaminados $\left(x^{2} ; p<0,001\right)$, así como sobre descarte de material punzocortante $(p=0,001)$. El descarte de guantes contaminados se hizo en bolsa de residuos comunes, tanto por parte de estudiantes de enfermería como de medicina. Los resultados evidencian que el conocimiento de los estudiantes sobre descarte de desechos infecciosos es débil e insuficiente para garantizar su aplicabilidad en la práctica.

Descriptores: Residuos Sanitarios; Administración de Residuos; Estudiantes de Enfermería; Estudiantes de Medicina; Occupational Exposure. 


\section{INTRODUÇÃO}

Resíduos de serviços de saúde (RSS) podem ser gerados em todos os ambientes onde são prestados cuidados em saúde ${ }^{(1)}$. Conforme sua periculosidade, esses resíduos são classificados, segundo a legislação brasileira, em cinco grupos: $A$ - infectantes (subdivididos em: $A 1$, A2, A3, A4 e A5), B- químicos, C - radioativos, D comuns, $\mathrm{E}$ - perfurocortantes ${ }^{(1-2)}$.

Essa classificação possibilita a segregação, que consiste na separação dos resíduos no momento de sua geração, para que os mesmos possam ser acondicionados, receber tratamento e destino final conforme os riscos que representam ${ }^{(3)}$.

Apesar da segregação ser determinante no manejo dos resíduos, muitos estudos apontam falhas nessa etapa ${ }^{(3-5)}$. Esses erros podem acontecer por falta de conhecimento e/ou conscientização ${ }^{(6-9)}$ e/ou precariedade de insumos adequados, como recipientes para acondicionamento de resíduos nos locais certos e deficiência de estrutura física ${ }^{(3-4)}$.

Os erros decorrentes da segregação ocorrem no descarte de resíduos de todos os grupos. Há resíduos comuns em sacos destinados aos infectantes ou em caixas de paredes rígidas. Há perfurocortantes em sacos e infectantes junto aos resíduos comuns ${ }^{(3-4)}$.

Essas falhas podem ocasionar riscos ocupacionais, principalmente ao desprezar perfurocortantes em locais inapropriados, desencadear danos ambientais ao dispor resíduos no meio ambiente sem tratamento prévio e elevar os custos econômicos, ao encaminhar para tratamento resíduos que não requeriam tal processo.

Destaca-se que o descarte inadequado de perfurocortantes representa uma das causas mais comuns de acidentes com material biológico. De março de 2012 a outubro de 2013 foram notificados 10.088 acidentes com material biológico em serviços de saúde brasileiros e, desses, mais de 1.400 (14,7\%) ocorreram pelo descarte inadequado de resíduos ${ }^{(10)}$. Estudos realizados com populações específicas também confirmam esses achados, sendo o descarte inadequado de perfurocortantes responsável por $15,6 \%$ a $21,0 \%$ das causas de acidentes ocupacionais com exposição a material biológico ${ }^{(11-14)}$.

A responsabilidade pelo descarte do resíduo é de quem o gerou ${ }^{(1)}$. Assim, todos os atores sociais envolvidos nesse processo - profissionais de saúde, estudantes da área de saúde, cuidadores e usuários - precisam conhecer e estar conscientes de seu importante papel no manejo dos resíduos.
O conhecimento do manejo dos RSS, pelos profissionais da área de saúde, é fundamental para favorecer a adequada segregação e a consequente proteção profissional e ambiental. Considerando que todos os profissionais geram resíduos, essa temática precisa ser problematizada desde o início dos cursos de graduação, antecedendo $o$ início das atividades práticas dos estudantes, com vistas à conscientização e mudança de comportamento.

Nesse contexto, este trabalho foi desenvolvido com acadêmicos de graduação em enfermagem e medicina, por desenvolverem atividades práticas que geram resíduos infectantes. Esses acadêmicos, em fase de conclusão de curso, estarão, em um futuro muito próximo, inseridos no mercado de trabalho, portanto, responsáveis pela segregação de materiais; alguns, possivelmente, estarão à frente do planejamento e gerenciamento dos resíduos em seus locais de atuação.

Apesar disso, há poucos estudos nacionais que abordam essa temática ${ }^{(15-16)}$ e uma escassez de trabalhos internacionais recentes que retratem o conhecimento dos acadêmicos de enfermagem e de medicina sobre o descarte de resíduos ${ }^{(17)}$.

Sendo assim, objetivamos verificar o conhecimento e a prática dos acadêmicos de enfermagem e de medicina a respeito do descarte de resíduos infectantes e perfurocortantes. Acreditamos que os resultados deste estudo trarão subsídios para as instituições formadoras reavaliarem o contexto da abordagem desta temática durante a graduação.

\section{MÉTODO}

Estudo transversal, descritivo, observacional, realizado com acadêmicos do último ano/período dos cursos de graduação em enfermagem e medicina de uma universidade pública de Goiás.

A coleta de dados ocorreu em duas etapas. $\mathrm{Na}$ primeira, foi aplicado um questionário aos estudantes dos dois cursos, com questões fechadas e abertas, cujo objetivo era verificar o conhecimento sobre o descarte de resíduos infectantes e perfurocortantes. Participaram dessa etapa todos os acadêmicos de enfermagem e de medicina do último ano dos cursos, presentes em sala de aula no dia da coleta de dados. Essa fase da coleta foi realizada pela pesquisadora principal após observação dos aspectos éticos.

Os instrumentos foram submetidos ao julgamento de sete juízes, quatro profissionais considerados peritos no ensino, pesquisa e assistência e ainda por outros três profissionais com ampla vivência na área assistencial em 
termos relacionados à infectologia, mais precisamente às medidas preventivas de infecções em estabelecimentos da área de saúde. Após análise, discussão das sugestões dos apreciadores e teste piloto, os instrumentos foram ajustados. O primeiro bloco do questionário continha questões que objetivavam caracterizar a população estudada: curso, data de nascimento, sexo e outros. As demais questões foram elaboradas de acordo com a resolução da ANVISA 306/2004(1) e abordavam aspectos sobre a segregação dos resíduos infectantes e perfurocortantes.

Na segunda etapa, os dados foram coletados por meio de observação direta dos acadêmicos nos locais de assistência do hospital (clínicas médica, cirúrgica, tropical, pediátrica, ginecológica, pronto-socorro, UTI clínica e cirúrgica) e registrados em uma lista de verificação com itens que permitiam identificar a forma como os acadêmicos descartavam os resíduos infectantes e perfurocortantes.

Todos os estudantes que responderam ao questionário, desenvolveram atividades práticas ou fizeram internato em unidades assistenciais do hospital de ensino durante o último ano de graduação, e que puderam ser observados em sua prática, constituíram a população de estudo na segunda etapa da investigação.

Os acadêmicos foram observados, pelo menos uma vez, durante as atividades de cuidar, as quais envolviam o descarte de resíduos. Para evitar interferência nos resultados, a pesquisadora que aplicou o questionário não atuou nessa fase da coleta dos dados. Participaram dessa etapa dez auxiliares de pesquisa previamente orientados e qualificados, visando à uniformidade dos dados. Eles foram posicionados de forma estratégica nas unidades assistenciais, a fim de facilitar a observação dos acadêmicos durante a execução dos procedimentos investigados. Optou-se por observar um acadêmico do início ao fim do procedimento, para só então iniciar a observação de outro. A postura discreta dos auxiliares de pesquisa durante a coleta foi cuidadosamente planejada.

Os dados foram analisados por meio de estatística descritiva utilizando frequência simples. Em seguida, foram processados pelo programa Sigma Stat ${ }^{\circledR}$, versão $2,03^{\circledR}$. Para verificar as variáveis categóricas, foram utilizados os testes de $x^{2}$ e Exato de Fisher, considerando $\mathrm{p}<0,05$.

O projeto de pesquisa foi aprovado pelo Comitê de Ética em Pesquisa do Hospital das Clínicas da Universidade Federal de Goiás, no 043/07. Os dados foram coletados após a anuência dos participantes pela assinatura do Termo de Consentimento Livre e Esclarecido, no momento da aplicação do questionário.

\section{RESULTADOS}

Responderam ao questionário 48 acadêmicos de enfermagem $(100,0 \%)$ e 93 de medicina $(83,0 \%)$. Foram observados em situações práticas 26 acadêmicos de enfermagem $(54,2 \%)$ e 78 de medicina $(69,6 \%)$. Não houve diferença estatística significante entre a população prevista e a estudada, $\mathrm{p}=0,333$.

Os acadêmicos de enfermagem e de medicina observados em situações práticas tiveram 45 e 20 oportunidades, respectivamente, de desprezar resíduos infectantes. Esse descarte ocorreu em saco para resíduo comum pelos acadêmicos de enfermagem em 37 oportunidades, enquanto os acadêmicos de medicina 0 fizeram em 16. Entre os resíduos infectantes descartados, destacam-se materiais de curativo e luvas contaminadas com agente biológico (Tabela 1).

Os acadêmicos de enfermagem tiveram 44 oportunidades de descartar luvas contaminadas com material biológico enquanto os de medicina tiveram 16 (Tabela 2).

Tabela 1: A prática do descarte de resíduo infectante em saco para resíduos comuns pelos acadêmicos de enfermagem ( $\mathrm{n}=$ 37)* e de medicina $(n=16) *$. Goiânia, GO, Brasil, 2007.

\begin{tabular}{|c|c|c|c|c|}
\hline \multirow{2}{*}{ Tipo de material infectante } & \multicolumn{2}{|c|}{ Acadêmicos de Enfermagem } & \multicolumn{2}{|c|}{ Acadêmicos de Medicina } \\
\hline & $\mathbf{n}$ & $\%$ & $\mathbf{n}$ & $\%$ \\
\hline Luvas & 12 & 32,4 & 06 & 37,5 \\
\hline Material de curativo & 19 & 51,3 & 04 & 25,0 \\
\hline Abaixador de língua & 00 & 0,0 & 03 & 18,0 \\
\hline Seringa & 02 & 5,4 & 00 & 0,0 \\
\hline Sonda & 02 & 5,4 & 00 & 0,0 \\
\hline Outros & 02 & 5,4 & 03 & 18,7 \\
\hline
\end{tabular}

* Refere-se ao número de observação de descarte por grupos de acadêmicos. 
Tabela 2: Respostas e ações realizadas pelos acadêmicos de enfermagem e de medicina quanto ao local de descarte de luvas contaminadas com material biológico. Goiânia, GO, Brasil, 2007.

\begin{tabular}{|c|c|c|c|c|c|c|}
\hline \multirow{2}{*}{ Local de descarte } & \multicolumn{2}{|c|}{ Acadêmicos de Enfermagem } & \multicolumn{2}{|c|}{ Acadêmicos de Medicina } & \multirow{2}{*}{ Teste } & \multirow{2}{*}{$\mathbf{p}$} \\
\hline & $\mathbf{n}$ & $\%$ & $\mathbf{n}$ & $\%$ & & \\
\hline Conhecimento & \multicolumn{2}{|c|}{$(n=48)$} & \multicolumn{2}{|c|}{$(n=93)$} & $\mathbf{x}^{2}$ & \\
\hline Saco branco leitoso & 43 & 89,6 & 49 & 52,7 & 19,176 & $<0,001$ \\
\hline Resíduo comum & 3 & 6,2 & 10 & 10,7 & 0,284 & 0,594 \\
\hline Desconhecem o tipo & 1 & 2,1 & 34 & 36,6 & 17,947 & $<0,001$ \\
\hline Não informado & 1 & 2,1 & 0 & 0 & - & - \\
\hline Prática & \multicolumn{2}{|c|}{$(n=44) *$} & \multicolumn{2}{|c|}{$(n=16) *$} & \multicolumn{2}{|c|}{ Exato de Fisher } \\
\hline Saco branco leitoso & 32 & 72,7 & 10 & 62,5 & \multicolumn{2}{|c|}{$-0,53$} \\
\hline Resíduo comum & 12 & 27,3 & 6 & 37,5 & & \\
\hline
\end{tabular}

* Refere-se ao número de observação de descarte por grupos de acadêmicos.

No total, 43 acadêmicos de enfermagem $(89,6 \%)$ e 49 de medicina $(52,6 \%)$ responderam que as luvas contaminadas com material biológico devem ser descartadas em saco branco leitoso. Na prática, em 32 $(72,7 \%)$ e em $10(62,5 \%)$ oportunidades, os acadêmicos de enfermagem e de medicina, respectivamente, descartaram luvas no saco apropriado, conforme Tabela 2.

A Tabela 3 apresenta as respostas fornecidas pelos acadêmicos dos dois cursos em relação ao descarte de resíduos perfurocortantes.

Tabela 3: Respostas fornecidas pelos acadêmicos de enfermagem $(n=48)$ e de medicina $(n=93)$ quanto ao descarte de perfurocortantes após o uso. Goiânia, GO, Brasil, 2007.

\begin{tabular}{|c|c|c|c|c|c|c|}
\hline \multirow[t]{2}{*}{ Variáveis } & \multicolumn{2}{|c|}{$\begin{array}{l}\text { Acadêmicos de } \\
\text { Enfermagem }\end{array}$} & \multicolumn{2}{|c|}{$\begin{array}{l}\text { Acadêmicos de } \\
\text { Medicina }\end{array}$} & \multirow[t]{2}{*}{$x^{2}$} & \multirow[t]{2}{*}{$\mathbf{p}$} \\
\hline & $\mathbf{n}$ & $\%$ & $\mathbf{n}$ & $\%$ & & \\
\hline \multicolumn{7}{|c|}{$\begin{array}{l}\text { Recipiente do descarte de PC deve ter parede } \\
\text { rígida }\end{array}$} \\
\hline Verdadeiro & 47 & 97,9 & 90 & 96,8 & 0,393 & 0,531 \\
\hline Falso & 0 & 0,0 & 03 & 3,2 & & \\
\hline Não informado & 01 & 2,1 & 0 & 0,0 & & \\
\hline \multicolumn{7}{|c|}{ PC devem ser deixados em bandeja após o uso } \\
\hline Verdadeiro & 05 & 10,4 & 78 & 83,9 & 66,368 & 0,001 \\
\hline Falso & 42 & 87,5 & 15 & 16,1 & & \\
\hline Não informado & 01 & 2,1 & 0 & 0,0 & & \\
\hline \multicolumn{7}{|c|}{$\begin{array}{l}\text { Recipiente de descarte deve estar próximo ao } \\
\text { local de procedimento }\end{array}$} \\
\hline Verdadeiro & 36 & 75,0 & 79 & 84,9 & 0,970 & 0,325 \\
\hline Falso & 11 & 22,9 & 14 & 15,0 & & \\
\hline Não informado & 01 & 2,1 & 0 & 0,0 & & \\
\hline
\end{tabular}

* PC - Perfurocortante

Em situações práticas, 12/12 (100,0\%) dos acadêmicos de enfermagem que desprezaram perfurocortantes o fizeram em recipiente apropriado. Descartaram também corretamente 06/07 (85,7\%) dos acadêmicos de medicina. Destaca-se que 01/07 (14,3\%) dos acadêmicos de medicina deixou o material utilizado dentro da bandeja.

\section{DISCUSSÃO}

Todos os profissionais devem se preocupar com os resíduos gerados durante suas atividades com o objetivo de minimizar riscos ao meio ambiente e à saúde dos trabalhadores, bem como da população em geral $^{(3,18)}$.

Neste estudo optamos por verificar o conhecimento e a prática do descarte de luvas contaminadas com material biológico e perfurocortantes, por entendermos que são os artigos mais utilizados pelos acadêmicos, o que facilitaria a observação do descarte e a comparação entre conhecimento e prática, tornando a análise mais fidedigna.

Observamos uma diferença estatisticamente significante quanto à demonstração de possuir informações a respeito do descarte de luvas contaminadas com material biológico apresentada pelos acadêmicos de enfermagem em relação aos de medicina (Tabela 2). Verificamos que $34(36,6 \%)$ dos acadêmicos de medicina afirmaram desconhecer o tipo de saco recomendado para descartar luvas contaminadas com material biológico. Estudo realizado em Minas Gerais evidenciou que 62,2\% dos estudantes de graduação de ciclos básicos das áreas biológicas e de saúde desconhecem a cor do saco recomendada para o acondicionamento de resíduos infectantes $^{(16)}$. Destacamos que o conhecimento do 
recipiente adequado para esse descarte é um dos requisitos básicos para a adequada segregação.

Os dados observados nas situações práticas mostram a dicotomia entre o conhecimento (43, o equivalente a $89,6 \%$ ) e a prática (12, o equivalente a $27,3 \%$ ) do descarte de luvas contaminadas pelos acadêmicos de enfermagem e confirmam o pouco conhecimento (49, que representa $52,7 \%$ ) demonstrado pelos graduandos de medicina sobre esse descarte.

Em estudo semelhante realizado com estudantes e docentes de medicina, medicina veterinária e enfermagem foi demonstrado que os profissionais de medicina não se envolvem com a geração dos resíduos. Os resultados revelaram ainda maior envolvimento da enfermagem no manejo dos RSS, por assumirem papéis de gestão de serviços, fato que leva outros profissionais a se sentirem desobrigados de tal função(19). Os estudantes percebem a falta de domínio e interesse do professor ao abordar a temática de RSS por não haver uma disciplina específica para esta questão, ficando a discussão do tema pontual, restrita a algumas horas e marcada pela fragmentação no ensino, que não articula a teoria com a prática ${ }^{(19)}$.

Os mesmos autores enfatizam que a problematização sobre o manejo dos resíduos desde o início da formação possibilitaria aos futuros profissionais maior compreensão e instrumentalização para o enfrentamento dessa relevante questão socioambiental ${ }^{(19)}$ e econômica.

Nossos resultados sugerem que o manejo de resíduos não é um conteúdo aprendido no curso de graduação em medicina. Provavelmente, os professores entendem que esta temática não seja de sua competência profissional e, portanto, não a abordam no processo educativo. Estudos precisam ser desenvolvidos para explicitar essa realidade. Embora tenha sido evidenciado o conhecimento dos acadêmicos de enfermagem sobre a temática, este não foi construído de forma conscientizadora, uma vez que não gerou mudanças na prática.

Há necessidade de enfatizar a questão do manejo dos resíduos de serviços de saúde durante a formação dos profissionais da área de saúde, uma vez que, por estarem relegados ao lixo, esses resíduos recebem pouca importância. No entanto, a conscientização e o efetivo manejo irão refletir em sustentabilidade ambiental e segurança ocupacional para além do circuito dos profissionais de saúde.

Apesar de não atuarem no gerenciamento dos resíduos, todos os profissionais que os geram devem saber como descartá-los, pois são responsáveis pela primeira etapa do manejo (a segregação) que, por sua vez, interfere nas demais.
A segregação é o ponto fundamental de toda discussão sobre a periculosidade ou não dos resíduos de serviços de saúde. Apenas uma parcela é potencialmente infectante, contudo, se ela não for segregada adequadamente, todos os resíduos que a ela estiverem misturados também deverão ser tratados como potencialmente infectantes. Isso exige procedimentos especiais para acondicionamento, coleta, transporte e disposição final, o que eleva os custos e riscos ocupacionais $^{(3-4)}$.

Em inventário realizado em uma faculdade de odontologia, foram pesados os resíduos potencialmente infectantes, da forma como segregados pelos profissionais e após a segregação realizada pelos pesquisadores. Houve redução de $80 \%$ dos resíduos potencialmente infectantes e, dentre aqueles descartados erroneamente, havia, inclusive, materiais que poderiam ser reciclados. A geração anual de resíduos dessa faculdade havia sido estimada em três toneladas e $80,0 \%$ do que foi descartado como grupo A pertencia, com a segregação adequada, ao grupo D (comum). Os dados mostraram que 2,4 toneladas de lixo/ano estão sendo encaminhadas para tratamento desnecessário no aterro sanitário(20). Destacamos que a origem deste problema está na segregação.

Estudo desenvolvido em serviços de urgência mostra realidade semelhante, onde aproximadamente $70,0 \%$ dos resíduos segregados como infectantes eram, de fato, resíduos comuns ${ }^{(3)}$. Essa mesma realidade também foi encontrada em nove unidades básicas de saúde no Brasil, nas quais apenas $34,1 \%$ dos resíduos descartados como infectantes pertenciam realmente a esse grupo. 0 restante eram resíduos comuns ou perfurocortantes descartados como infectantes ${ }^{(21)}$.

O gerenciamento adequado dos resíduos de serviços de saúde pode contribuir, significativamente, para a redução da ocorrência de acidentes de trabalho, especialmente aqueles provocados por perfurocortantes. Estudos $^{(22-24)}$ mostram que a maioria dos acidentes com perfurocortantes ocorre durante o descarte e, principalmente, pelo descarte inapropriado.

Evidenciamos que os acadêmicos de enfermagem apresentaram conhecimento mais consistente, com uma diferença estatisticamente significativa $(p=0,001)$, quanto ao conhecimento sobre o local de descarte dos perfurocortantes. Estudo ${ }^{(16)}$ desenvolvido com alunos dos ciclos básicos de ciências biológicas e da saúde constatou que $75,5 \%$ não possuíam informação suficiente para lidar com os materiais e resíduos perfurocortantes e escarificantes gerados nas práticas laboratoriais. 
A prática do descarte de resíduos do grupo $\mathrm{E}$ ocorreu de forma adequada por todos os acadêmicos de enfermagem. Os de medicina, além de afirmarem que os perfurocortantes utilizados devem ser deixados em bandeja $78(83,9 \%)$ representando uma diferença estatisticamente significante $(p=0,001)$, foram os que, na prática $(14,3 \%)$, os descartaram na bandeja. Ao contrário do encontrado no nosso estudo, pesquisa desenvolvida na Província de Mazandaran, no Iran, a respeito do conhecimento e prática de profissionais de saúde e estudantes de medicina sobre as precauções padrão, verificou boas práticas no descarte de resíduos perfurocortantes $^{(17)}$.

Não encontramos mais estudos que mostrem esse tipo de descarte por acadêmicos de medicina em outros locais. Porém, o descarte inadequado é uma prática comum do staff médico no locus pesquisado, o que evidencia a importância do modelo profissional na solidificação do conhecimento do graduando.

Este dado é preocupante, pois além de refletir o não conhecimento dos acadêmicos em relação à temática, também nos alerta para o fato de que, ao pensarem e agirem dessa maneira, estão expondo a si e aos outros a risco de acidentes com material biológico.

A prática adequada do manejo de perfurocortantes e a segregação dos resíduos infectantes na fonte geradora constituem necessidades urgentes e fundamentais para o gerenciamento de riscos e recursos. Autores ${ }^{(25)}$ referem que o conhecimento dos custos associados ao uso de materiais e insumos, e ao seu tratamento após utilização, pode reduzir o uso descontrolado e inadequado de materiais hospitalares. A expectativa é que profissionais de todas as categorias e áreas de atuação, conscientes da importância da correta segregação dos resíduos gerados nos serviços de saúde, sejam mais atuantes no processo,

\section{REFERÊNCIAS}

1. Ministério da Saúde. Agência Nacional de Vigilância Sanitária. Resolução da Diretoria Colegiada da Anvisa número 306 de 7 de dezembro de 2004 (BR). Dispõe sobre o tratamento e disposição final dos resíduos de serviços de saúde. Diário Oficial da União (Brasília). 2004 dez 10.

2. Ministério do Meio Ambiente. Resolução do Conselho Nacional do Meio Ambiente número 358 de 29 de abril de 2004. Dispõe sobre o tratamento e a disposição final dos resíduos de serviços de saúde e dá outras providências. Diário Oficial da União (Brasília). 2005 mai 04. 3. Pereira MS, Alves SB, Souza ACS, Tipple AFV, Rezende FR, Rodrigues EG. Waste management in non-hospital emergency units. Rev Lat Am Enfermagem.2013; 21(spe): 259-66. encaminhando para tratamento apenas aqueles materiais que realmente necessitem ser tratados.

O descarte dos resíduos é responsabilidade de quem os gera; assim, o desconhecimento do tema, pelos acadêmicos, torna-se um desafio tanto para os estabelecimentos de ensino quanto para os de saúde, com reflexos nocivos para trabalhadores e meio ambiente.

Salientamos como limitações deste estudo o fato de não terem sido observadas as questões de infraestrutura relacionada ao descarte dos resíduos e que nem todos os acadêmicos que responderam ao questionário puderam ser observados na prática, o que impossibilitou uma comparação pareada entre conhecimento e prática. Além disso, em função dos locais de realização da coleta de dados, não foi possível observar o descarte dos resíduos infectantes pertencentes a todos os subgrupos.

\section{CONCLUSÃO}

Os acadêmicos de enfermagem apresentaram conhecimento mais consistente do que os de medicina ( $p=$ 0,001 ) sobre a indicação do local de descarte de luvas contaminadas e no que se refere à recomendação de não deixar perfurocortantes em bandejas, após o uso.

O conhecimento dos acadêmicos de enfermagem e medicina a respeito do descarte de resíduos infectantes e perfurocortantes revelou-se frágil pela sua inconsistência, visto não ter sido construído de forma suficiente para garantir sua aplicabilidade na prática.

É necessário rever o conteúdo e a forma como tem sido tratada a questão do manejo dos resíduos de serviços de saúde durante a graduação, especialmente no curso de medicina. A compreensão de que o manejo dos resíduos é responsabilidade de todos e uma questão de saúde pública é fundamental para a formação de profissionais comprometidos com a saúde ocupacional, segurança do paciente, saúde pública e meio ambiente.

4. Alves SB, Souza ACS, Tipple AFV, Rezende KCD, Rezende FR, Rodrigues EG. Manejo de resíduos gerados na assistência domiciliar pela estratégia saúde da família. Rev Bras Enferm. 2012; 65(1):128-34.

5. Mazrui, N. Medical waste management in Kenya: opportunities for improvement. Tropical resources. Tropical resources. 2010;29 (s/n):66-70.

6. Tenglikar PV, Kumar GA, Kapate R, Reddy S, Vijayanath $\mathrm{V}$. Knowledge attitude and practices of health care waste management amongst staff of nursing homes of Gulbarga city. J Pharm Biomed Sci. 2012; 19(12):13.

7. Archisman M, Gupta Manoj K, Shivalli S, Mishra CP, Mohapatra SC. Biomedical waste management practices of doctors: an online snapshot. National Journal of Community Medicine. 2012; 3(2):227-31. 
8. Atif ML, Brenet A, Hageaux S, Fave $M H$, Cochet $\mathrm{C}$, Baticle $\mathrm{E}$ et al. Awareness of standart precautions for 4439 health care professionals in 34 institutions in France. Medecine et maladies infectieuses. 2013;43:1016.

9. Sharma A, Sharma V, Sharma S, Singh P. Awareness of biomedical waste management among health care personnel in Jaipur, India. Oral Health Dent Manag. 2013;12(1):32-40.

10. Risco Biológico. Relatório PSBIO - Sistema de Vigilância de Acidentes de Trabalho com material biológico em serviços de saúde brasileiros. Rio de Janeiro, 2013.

11. Ramos CM, Santos RIM. Acidente de trabalho com exposição à material biológico e o anti-hbs como resposta imunológica à vacina contra hepatite B. Omnia Saúde. 2009:6(2):15-28.

12. Valim MD, Marziale MHP. Avaliação da exposição ocupacional a material biológico em serviços de saúde. Texto Contexto Enferm. Florianópolis.

$2011 ; 20$ (Esp):138-46.

13. Dias MAC, Machado AA, Santos BMO. Acidente ocupacional com exposição à material biológico: retrato de uma realidade. Medicina (Ribeirão Preto).

2012;5(1):12-22.

14. Araujo TM, Caetano JA, Barros LM, Lima ACF, Costa RM, Monteiro VA. Acidentes de trabalho com exposição a material biológico entre profissionais de enfermagem.

Rev Enf Ref. 2012;III(7):7-14.

15. Moreschi C, Rempel C, Backes DS, Carreno I, Siqueira DF, Marina B. A importância dos resíduos de serviços de saúde para docentes, discentes e egressos da área da saúde. Rev Gaucha Enferm. 2014;35(2):206. 16. Stehling MC, Cunha LM, Louredo LM, Camargo CG, Haddad JPA, Silva IJ, et al. Gestão de resíduos com risco biológico e perfurocortantes: conhecimento de estudantes de graduação das áreas biológicas e da saúde da Universidade Federal de Minas Gerais. Rev Min Enferm. 2013;17(3):594-600.

17. Motamed N, BabaMahmoodi F, Khalilian A, Peykanheirati M, Nozari M. Knowledge and practices of health care workers and medical students towards universal precautions in hospitals in Mazandaran Province. East Mediterr Health J. 2006;12(5):653-61. 18. Costa TF, Felli VE, Baptista PCP. A percepção dos trabalhadores de enfermagem sobre o manejo dos resíduos químicos perigosos. Rev Esc Enferm USP. 2012;46:1453-1461.

19. Corrêa LB, Lunardi VL, De Conto SM. O processo de formação em saúde: o saber resíduos sólidos de saúde em vivências práticas. Rev Bras Enferm. 2007; 60(1):215.

20. Paiva EMM, Tipple AFV, Sasamoto SA. Plano de

Gerenciamento de resíduos de serviços de saúde PGRSS2007 da Faculdade de Odontologia da UFG. 2007; Goiânia: UFG/Faculdade de Odontologia; 2007.

21. Alves SB, Souza ACS, Tipple AFV, Rezende KCAD, Resende FR, Rodrigues EG, Pereira MS. The reality of waste management in primary health care units in Brazil. WM\&R. 2014; spe:1-8.

22. Oliveira AC, Paiva MHRS. Analysis of occupational accidents with biological material among professionals in pre-hospital services. Rev Lat Am Enfermagem. 2013; 21(1):309-15.
23. Vieira M, Padilha MI, Pinheiro RDC. Analysis of Accidents with Organic Material in Health Workers. Rev Lat Am Enfermagem. 2011;19(2):332-39.

24. Lopes LP, Toffano SEM, Hayashida M, Canini SRMS, Cruz EDA, Reinato LAF et al. Exposições acidentais com material biológico potencialmente contaminado envolvendo graduandos de enfermagem do último ano. Rev. Eletr. Enf. [Internet]. 2011 [acesso em: 18 junho 2013] out/dez;13(4):751-7. Disponível em: http://www.fen.ufg.br/revista/v13/n4/v13n4a21.htm. 25. Macedo LC, Larocca LM, Chaves MMN, Perna PO, Muntsch SMA, Damaceno EFC, et al. Segregação de resíduos nos serviços de saúde: a educação ambiental em um hospital-escola. Cogitare Enferm. 2007;12(2):183-8.

Artigo recebido em 28/06/2013. Aprovado para publicação em 10/09/2014. Artigo publicado em 31/03/2015. 